\title{
Mechanisms of Covalent Dimerization on a Bulk Insulating Surface
}

\author{
Chunyan Guo, ${ }^{\dagger}$ Yu Wang, ${ }^{*}{ }^{\dagger}$ Markus Kittelmann, ${ }^{\ddagger}$ Lev Kantorovitch, ${ }^{*}$, Angelika Kühnle, ${ }^{\ddagger}$ \\ and Andrea Floris*, $\|_{\odot}$ \\ ${ }^{\dagger}$ School of Physics and Technology, Wuhan University, Wuhan 430072, China \\ ${ }^{\ddagger}$ Institute of Physical Chemistry, University of Mainz, 55099 Mainz, Germany \\ ${ }^{\S}$ Department of Physics, King's College London, Strand, London WC2R 2LS, United Kingdom \\ "School of Mathematics and Physics, University of Lincoln, Brayford Pool, LN6 7TS Lincoln, United Kingdom
}

Supporting Information

\begin{abstract}
Combining density functional theory and highresolution NC-AFM experiments, we have studied the onsurface reaction mechanisms responsible for the covalent dimerization of 4-iodobenzoic acid (IBA) organic molecules on the calcite (10.4) insulating surface. When annealed at 580 $\mathrm{K}$, the molecules assemble in one-dimensional chains of covalently bound dimers. The chains have a unique orientation and result from a complex set of processes, including a nominally rather costly double dehalogenation reaction followed by dimerization. First, focusing on the latter two processes and using the nudged elastic band method, we analyze a number of possible mechanisms involving one and two molecules, and we isolate the key aspects facilitating the

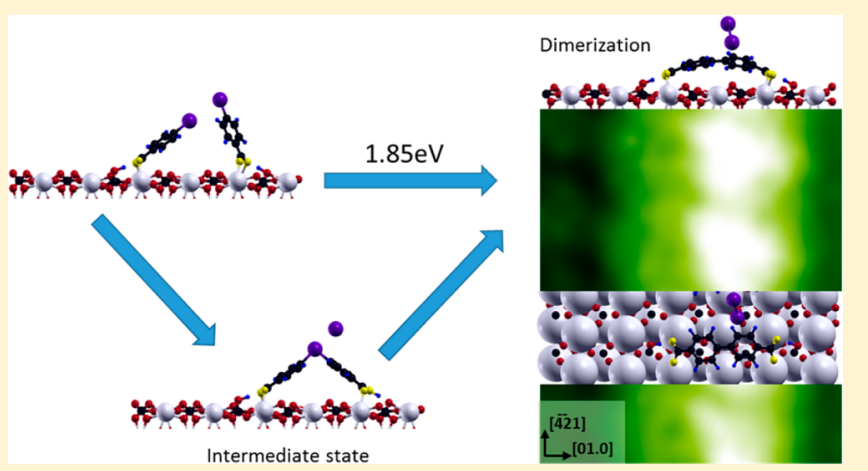
reaction on calcite. Second, we find that the insulating surface plays an active role as a catalyst by identifying two relevant processes: one exhibiting an intermediate state of chemisorbed molecules after independent dehalogenations and a second, highly nontrivial exothermic reaction channel in which two iodine atoms "cooperate" to minimize the cost of their individual detachment from the molecules. Both processes have dramatically reduced energy barriers compared to all other mechanisms analyzed. The knowledge of the formation mechanisms of a covalent assembly on insulators represents an important step toward the realization and control of structures that combine the robustness of covalent architectures with their electronic decoupling from the insulating substrate. This step has potentially important technological applications in nano- and molecular electronics.
\end{abstract}

\section{INTRODUCTION}

On-surface synthesis is a rapidly growing research field whose goal is the bottom-up fabrication of covalent nanostructures through the coupling of molecular building blocks ${ }^{1-7}$ (see also ref 8 and references therein). With this strategy, reaction products can be obtained under ultrahigh-vacuum conditions without using solvents and thus avoiding problems related to the lack of solubility. ${ }^{6}$ Moreover, the surface catalytic properties and molecular confinement resulting from the presence of a two-dimensional support open the possibility of new reaction pathways toward products not easily obtainable in three dimensions (i.e., in the gas phase or in solutions). ${ }^{6}$

Covalent nanostructures are quite robust in comparison with more "fragile" supramolecular architectures mediated by weaker interactions (hydrogen bonds, van der Waals, and electrostatic $^{9-15}$ ), in which the self-assembly is inherently linked to reversible interactions. From the technological point of view, the ability to control the synthesis of covalent nanostructures on a surface is crucial for their use in molecular devices operating in a wide range of physical conditions. Importantly, the use of insulating surfaces also guarantees the electronic decoupling between the support and the covalent nanostruc- tures. This makes the latter particularly suitable for electronic transport and, in general, for molecular electronic applications such as molecular nanodevices, ${ }^{6}$ molecular optics, ${ }^{16}$ electronics, and catalysis. ${ }^{17}$

These undoubtedly attractive technological perspectives have recently stimulated many experiments involving combinations of a large variety of molecular architectures on different substrates $^{1-4,6,18-25}$ (see also ref 8 and references therein).

Several experimental strategies to synthesize nanostructures on metal surfaces have proved very successful. These include approaches that exploit Ullmann-type processes ${ }^{2,6,22,23}$ (see also ref 8 and references therein) also studied in density-functionaltheory- (DFT-) based theoretical studies; ${ }^{8,26}$ strategies exploiting the activation of specific $\mathrm{C}-\mathrm{H}$ bonds, with the realization of very selective structures ${ }^{7,21,27,28}$ that in some cases have been rationalized by $\mathrm{DFT}^{5}$ and the use of terminal alkynes $^{6,8}$ or cyclodehydrogenation reactions, ${ }^{8,25}$ both analyzed theoretically. ${ }^{29-31}$ These achievements have been reviewed in

Received: March 21, 2017

Revised: April 11, 2017

Published: April 12, 2017 
refs 6 and 8 , focusing on the relevant experimental and theoretical studies to date, respectively.

Despite the considerable progress achieved on metal surfaces, comparatively fewer studies of synthesis on insulating substrates are available in the literature, including reactions on bulk surfaces through Ullmann-type processes, ${ }^{3,6,19,32}$ photochemical initiation, ${ }^{20}$ and reactions on insulating thin films ${ }^{18}$ (see also ref 6 and references therein). Several factors make on-surface syntheses on insulators rather challenging. ${ }^{3}$ First, these substrates cannot be investigated with techniques commonly used for conductive surfaces [e.g., scanning tunneling microscopy (STM), low-energy electron diffraction (LEED), and photoelectron spectroscopy (PES)]. Second, insulating supports are generally weakly reactive, ${ }^{3,33-36}$ because of a weak hybridization with molecular orbitals (leading to much lower binding energies), a lower molecule-surface van der Waals interaction, and their much lower surface energies compared to metallic surfaces. ${ }^{3,34}$ These facts might result in molecular desorption (before the reaction takes place) and dewetting effects, ${ }^{34}$ preventing the surface functionalization.

An important example of a reactive insulating substrate allowing the assembly of organic molecules is the calcite (10.4) surface. ${ }^{19,32,37-42}$ Its reactivity is due to an exceptionally high surface energy ${ }^{3}$ and to the presence of highly localized charges $^{34}$ as active sites suitable for molecular anchoring. ${ }^{3,43}$

A number of covalently linked structures have been obtained experimentally on calcite (10.4). ${ }^{6}$ Use of 4-iodobenzoic acid (IBA) molecules resulted in the formation of molecular dimers that assemble into one-dimensional chains of side-by-side aligned dimers. ${ }^{4}$ More complex molecules resulted instead in zigzag and closed-ring structures by sequential dehalogenations. ${ }^{19}$ These architectures were obtained by Ullmann-type or hierarchical sequential linking reactions. However, to the best of our knowledge, there are no theoretical works on the mechanisms of molecular synthesis on insulators providing insights into the hierarchy of possible reaction pathways, although a related work on boron nitride and graphene on $\mathrm{Ni}(111)$ was reported. ${ }^{46}$

In this work, we rationalize the covalent dimerization mechanisms of IBA molecules within one-dimensional chains observed in noncontact atomic force microscopy (NC-AFM) experiments ${ }^{4}$ on calcite (10.4) surface when annealed to $580 \mathrm{~K}$. We investigate the role of the surface in the dimerization process, considering whether it is chemically active or acts only as a two-dimensional support to constrain the molecules on a plane, a necessary condition for them to react. Focusing on the conditions making the cleavage of the iodine-phenyl bond feasible, we also address the validity of previous speculations ${ }^{4,19}$ that the cleavage of this bond could be facilitated by the deprotonation of the IBA carboxylic group on calcite. On the basis of a detailed analysis involving DFT and the nudged elastic band (NEB) method, ${ }^{44}$ we propose exothermic mechanisms for the dehalogenation-dimerization process (whereas the single dehalogenation in the gas phase is strongly endothermic). More importantly, we identify specific nontrivial mechanisms whereby the energy barrier is strongly reduced, rationalizing the experimental observations performed at the relevant temperatures.

\section{RESULTS AND DISCUSSION}

2.1. Experimental Section. The IBA molecule and the calcite (10.4) surface are presented in Figure 1. Our theoretical analysis starts from previous experimental studies based on NC-

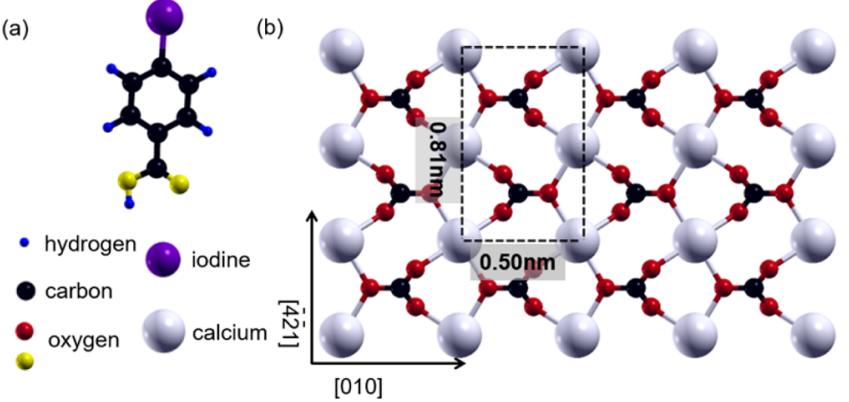

Figure 1. (a) Structure of an IBA molecule in the gas phase. (b) Top layer of the calcite (10.4) surface. The rectangular unit cell (shown by the dashed line) consists of two inequivalent $\mathrm{Ca}$ ions and two carbonate groups. The latter are rotated by $180^{\circ}$ with respect to each other. For better clarity, oxygen atoms belonging to the molecule and the surface are indicated in yellow and red, respectively.

AFM measurements. ${ }^{4}$ The latter showed that, upon molecular deposition on the substrate, a sequence of molecular phases is obtained during gradual annealing of the system. ${ }^{4}$ When deposited onto a calcite (10.4) surface held at room temperature, molecular islands form at substrate step edges. The islands exhibit a highly ordered inner structure as seen in Figure 2a, where we show a magnification of it. Upon annealing

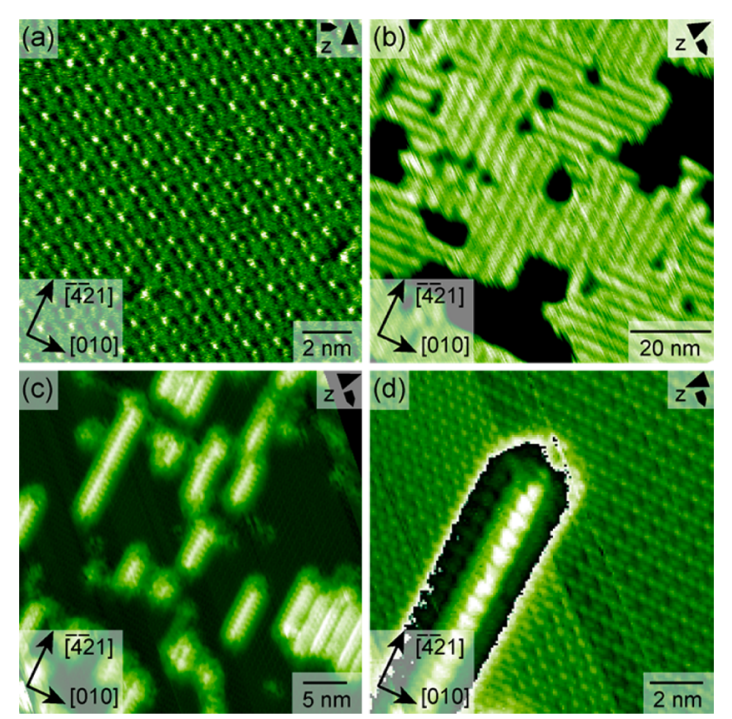

Figure 2. Three arrangements of IBA molecular structures on calcite (10.4) as a function of the sample temperature, obtained by NC$\mathrm{AFM}^{39}$ (a) highly ordered inner structure of molecular islands formed at room temperature, (b) upright phase observed after a moderate annealing to $520 \mathrm{~K}$, and $(\mathrm{c}, \mathrm{d})$ chains of dehalogenated IBA dimers arranged along the $[\overline{42} 1]$ direction obtained after annealing to $580 \mathrm{~K}^{4}$

at a moderate temperature of $520 \mathrm{~K}$, a first structural change is observed. From the apparent height of $0.8 \mathrm{~nm}$, we tentatively assign the molecules in this structure as adopting an upright configuration, ${ }^{32}$ denoted in the following as the upright phase. These upright-standing molecules form islands that are shown in Figure $2 \mathrm{~b} .{ }^{32}$ Upon annealing to $580 \mathrm{~K}$, a second structural change is observed. This structural change has previously been assigned to dehalogenation and dimerization of two IBA molecules to form biphenyl-4,4'-dicarboxylic acid (BPDCA, referred to for simplicity as "dimer" in the remainder of this work). ${ }^{4,39}$ The dimers arrange in chains oriented along the 


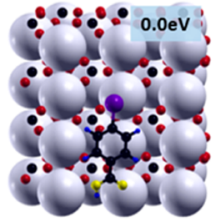

(a)

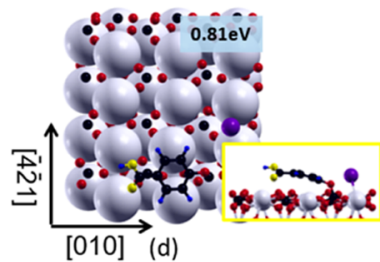

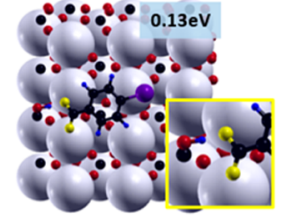

(b)

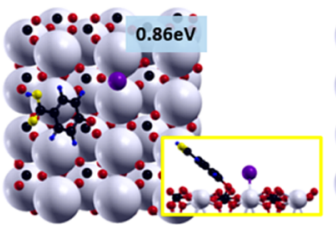

(e)

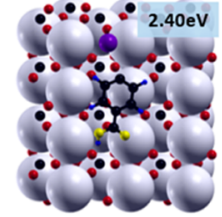

(c)

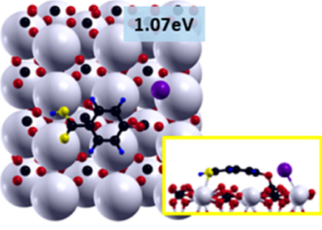

(f)

Figure 3. Flat-lying adsorption geometries of a single IBA molecule on calcite (10.4) in its (a,b) intact and (c-f) dehalogenated (de-Ha) configurations. (a) Most stable structure found, with its total energy set to $0 \mathrm{eV}$ as a reference for the energies of other configurations. The molecular axis forms an angle of $9^{\circ}$ with respect to the $\left[\overline{42} 1\right.$ ] direction. (b) Slightly less stable geometry forming a $19^{\circ}$ angle with respect to the [010] direction. The $\mathrm{cg}-\mathrm{H}$ atom relaxes at a position closer to the s-O than to the IBA carboxylic group oxygen (cg-O) (see inset), so that the roles of the s-O and cg$\mathrm{O}$ are interchanged, with the cg- $\mathrm{H}$ forming a $\mathrm{H}$-bond with the IBA (calculated $\mathrm{cg}-\mathrm{H}-\mathrm{cg}-\mathrm{O}$ distance of $\sim 1.4 \AA$ ). The two cg-O atoms bind with the two nearest $\mathrm{Ca}$ ions along [ $\overline{42} 1]$. To achieve this configuration, the protruding s-O must be available nearby for the cg- $\mathrm{H}$ to bind, with a concomitant breaking of the substrate $\mathrm{O}-\mathrm{Ca}$ bond. Placing the cg-H far away from the molecule (but still bound to the surface in an equivalent site) implies an energy cost of $>0.75 \mathrm{eV}$, which corresponds to the H-bond breaking (see section S5). (c) Unstable de-Ha structure with the phenyl radical not directly bonded to a surface atom. (d,e) Most stable de-Ha configurations with the flat phenyl radical bound to a calcite s-O atom. (f) Third lowenergy de-Ha structure also forming a bond between the carboxylate group and the surface Ca atom, with a concomitant IBA deformation.

$[\overline{42} 1]$ direction, as shown in Figure $2 \mathrm{c}^{39}$ A magnified image of one of these chains, shown in Figure $2 \mathrm{~d}$, suggests that it is composed of side-by-side aligned dimers (note that the atomic structure of the underlying calcite surface is visible as well). The measured apparent height of the molecules forming the chains $(0.3 \mathrm{~nm}$, much smaller than that for the upright phase, $0.8 \mathrm{~nm})$ and the thickness of each chain (compatible with two surface unit cells along [010]) strongly suggest that the chains are formed by flat-lying dimers. Here, the two molecules within each dimer are connected through a covalent bond between two carbon atoms that is formed upon a dehalogenation reaction. ${ }^{4,39}$

2.2. IBA Molecule in the Gas Phase. We start our theoretical analysis by simulating a single IBA molecule in the gas phase. The calculated energy cost to dissociate it by removing its iodine atom is $3.19 \mathrm{eV}$ (including two spin channels; see Supporting Information, section S1), compatible with the values to dehalogenate a different but related molecule (iodobenzene) measured experimentally $\left(2.79 \mathrm{eV}^{45}\right)$ and calculated theoretically $(3.33 \mathrm{eV})$ in a recent DFT study. ${ }^{26}$ This energy, which in the gas phase also coincides with the corresponding energy barrier, is far too high for the reaction to occur on the surface in the relevant interval of annealing temperatures $(520-580 \mathrm{~K}){ }^{53}$ unless a specific reaction mechanism that strongly reduces the barrier is at play. Our goal was to investigate the details of this dehalogenationdimerization reaction, analyzing the conditions under which it can occur and, hence, rationalizing the observed covalent assembly on calcite (10.4).

2.3. Simulated Systems. It can be speculated that the upright-to-chains phase transition consists of several distinct elementary processes. An exhaustive $a b$ initio analysis of the associated complex energy landscape is rather difficult, as no experimental information on the kinetics is available that would suggest "reasonable" intermediate states to model. Still, the dehalogenation reaction must be an elementary part of the whole transition, and hence, a mechanism leading to a reasonably small energy barrier for this reaction must exist.
After making the plausible assumption that the dehalogenation-dimerization reaction has the largest barrier of all elementary processes and "dominates" the phase transition, we focus our analysis only on this process. To this end, we consider only systems made of one single monomer on the surface, or a dimer with its axis oriented along [010], or a single chain of equivalent dimers periodically repeated along [ $\overline{42} 1]$.

2.4. Single Monomers on Calcite (10.4). Before identifying the factors that might lower the reaction barrier, we show in Figures 3 and 4 the adsorption geometries of a single monomer on the surface. We considered a large number $(\sim 20)$ of flat-lying intact (Figure 3a,b), flat-lying dehalogenated (Figure $3 \mathrm{c}-\mathrm{f}$ ), and upright relaxed configurations (Figures $4 \mathrm{a}-$ c). A detailed analysis is presented in sections S2 and S3. The most stable structure of a single molecule is presented in Figure $3 \mathrm{a}$, showing a flat molecule on the surface with a calculated adsorption energy of $1.53 \mathrm{eV}$. All energies provided in Figures 3 and 4 refer to the total energy of this structure. A second, slightly less stable but differently oriented configuration is shown in Figure $3 b$, where we show in the inset that the hydrogen atom of the carboxylic group (denoted $\mathrm{cg}-\mathrm{H}$ ) relaxes to a position closer to the nearest surface oxygen ( $\mathrm{s}-\mathrm{O}$ ), away from the IBA carboxylic group oxygen (cg-O). In other words, the cg-H does not detach completely but still binds to the parent molecule through a hydrogen bond, similarly to the case of a 2,5-dihydroxybenzoic acid (DHBA) molecule on the same substrate. $^{43}$

As for the IBA upright configurations, the structure in Figure 4a shows the cg-H still attached to the molecule as in the gas phase, whereas in the almost-degenerate geometries in Figure $4 \mathrm{~b}, \mathrm{c}$, a H-bond similar to that in Figure $3 \mathrm{~b}$ is realized. The fact that the structures in Figures $3 \mathrm{a}, \mathrm{b}$ and $4 \mathrm{a}-\mathrm{c}$ are almost degenerate indicates that the slight $\mathrm{cg}-\mathrm{H}$ detachment can easily occur at finite temperatures and that the $\mathrm{cg}-\mathrm{H}$ atom might shuttle from one state to another as a result of thermal effects. ${ }^{54}$

Relevant dehalogenated (de-Ha) configurations are presented in Figure $3 \mathrm{c}-\mathrm{f}$, with total energies substantially larger than those of the intact molecules. The most stable are shown 

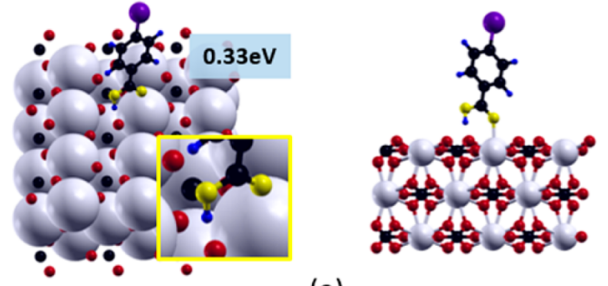

(a)
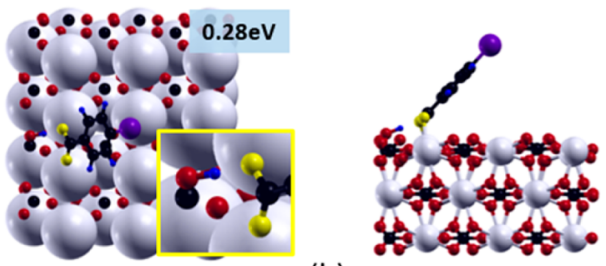

(b)
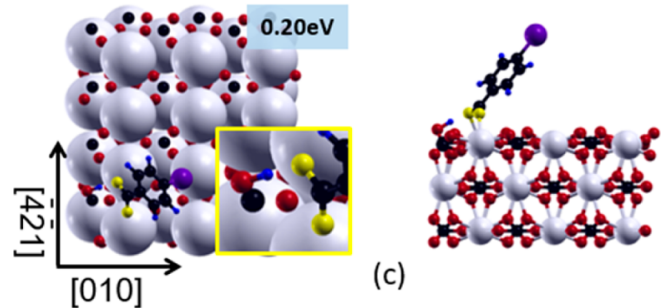

(c)

Figure 4. Upright adsorption geometries of a single IBA molecule on calcite (10.4). Left (right) panels give the top (side) views. (a) Geometry with the cg-H attached to the molecule. $(b, c)$ Slightly more stable geometries, in which the cg-H is slightly detached from the IBA and forms a H-bond with it (cf. the mechanism mentioned in the caption to Figure 3 and in ref 43). The total energies shown by the structures in the left panels are relative to the energy of the most stable structure of Figure $3 a$.

in Figure $3 \mathrm{~d}-\mathrm{f}$, where, upon dehalogenation, the molecule chemisorbs $^{52}$ and binds to the surface through its phenyl radical. The structure in Figure $3 c$ (and similar ones presented in section S3), in which this $\mathrm{C}-\mathrm{s}-\mathrm{O}$ bond is not formed, is considerably more unstable than the structures in Figure 3d,e (by $>1.6 \mathrm{eV}$ ) and Figure $3 \mathrm{f}$ (by $>1.3 \mathrm{eV}$ ).

2.5. Modeling IBA Dimers and Chains on Calcite (10.4). The final state of the reaction analyzed in this work has a dimeric structure, which we investigated by performing several DFT relaxations of conceivable IBA dimers within the chains (Figure 5). One possible dimerization is realized through a $\mathrm{C}-\mathrm{C}$ covalent bond upon a double dehalogenation (Figure $5 a)$. The second is realized through $\mathrm{H}$-bonds between the carboxylic groups of two IBA molecules (similarly to the DHBA molecule dimers in ref 43). We modeled only dimer configurations along [010], compatible with the experimental observations. The covalent dimer is energetically more stable than the $\mathrm{H}$-bonded one (by $1.23 \mathrm{eV}$ ), which is also substantially larger than the first (by $0.54 \mathrm{~nm}$ ). Hence, our calculations support the experimental conclusions that the chains are made of covalent dimers, ${ }^{4}$ and consequently, configurations similar to the one in Figure 5a are considered as the final product of the reaction studied in this work.

These single-molecule and dimer geometries provide crucial preliminary information for computing the energy barriers characterizing single and double de-Ha processes, followed by dimerization. In the following sections, we use the NEB method to understand how the effective barrier reduction can occur by considering several possible scenarios. A comparison

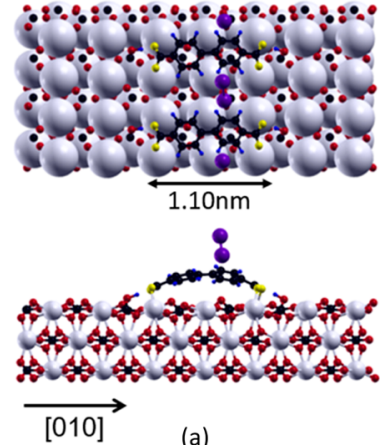

(a)

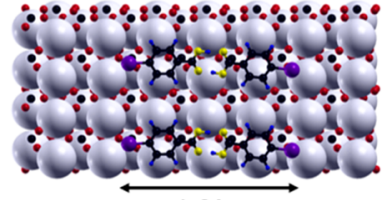

$1.64 \mathrm{~nm}$

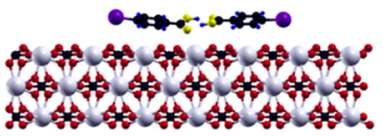

(b)
Figure 5. DFT relaxed chains of dimers on calcite (10.4) (top and side views). (a) Chain of covalent dimers made of dehalogenated IBA molecules connected through $\mathrm{C}-\mathrm{C}$ links (recombined $\mathrm{I}_{2}$ molecules are also present in the cell). The side view shows an arc-shaped structure due to a slight mismatch between the length of two calcite unit cells along [010] and that of the dimer $(1.10 \mathrm{~nm})$. The dimer deforms to establish the $\mathrm{Ca}-\mathrm{O}$ bonds with the surface, a stabilizing factor on the surface. Note that both the $\mathrm{H}$ atoms of the carboxylic group ( $\mathrm{cg}-\mathrm{H}$ atoms) move from the molecules toward the nearest surface oxygen atoms (s-O atoms), although we found that the corresponding structures in which the $\mathrm{cg}-\mathrm{H}$ atom(s) remain attached to the molecule(s) are degenerate in energy (see section S6). (b) Chain of dimers made of two intact molecules connected through double $\mathrm{H}$-bonds at the respective carboxylic groups. This chain is by $1.23 \mathrm{eV}$ less favorable (per dimer) than that in panel a and its size goes well beyond two unit cells along [010].

of the corresponding energy barriers guides the identification of the most probable reaction mechanism.

2.6. Independent de-Ha's Involving Flat-Lying Molecules. In the first scenario, we assume that two molecules dehalogenate independently and subsequently diffuse toward each other to form a dimer. Given the substantial equivalence of the two de-Ha reactions, only one barrier involving a single IBA molecule needs to be calculated. The most favorable process found among the set of reactions studied (see section S7) is illustrated in Figure 6. The IBA molecule is chemisorbed, with the phenyl radical bound to the surface. The associated barrier is $1.76 \mathrm{eV}, 1.43 \mathrm{eV}$ lower than the gas-phase value (3.19 $\mathrm{eV})$. The reduction is due both to the chemisorption and to the adsorption energy of iodine on the surface (clearly missing in the gas phase), which we calculated separately as $0.81 \mathrm{eV}$ (see section S4). This results in a much lower final-state (FS) energy, which, in turn, lowers the barrier itself. Total energy comparisons indicate that the FS of a chemisorbed de-Ha IBA on calcite would be less stable than the initial state (IS) of the intact molecule. This is in contrast to analogous processes on noble-metal surfaces, where dehalogenated iodobenzene and bromobenzene were calculated to have lower total energies than the respective intact molecules. ${ }^{26}$ Dehalogenation processes of this kind occur independently for each molecule. Their energy cost is clearly reduced by the surface (with respect to the gas phase). These reactions must be followed by diffusion of the de-Ha molecules and their dimerization, provided that they find themselves at a short distance. Iodine atoms might also diffuse and recombine to form $\mathrm{I}_{2}$. Experimentally, there is no evidence for iodine atoms or $\mathrm{I}_{2}$ molecules adsorbed on the surface. However, one could easily envision that $\mathrm{I}_{2}$ desorbs and goes to the gas phase, a process that has an associated cost of $0.78 \mathrm{eV}$ (see section S4). Given that the reactions are occurring at high temperatures, changes in the free energy rather than the total energy must be 


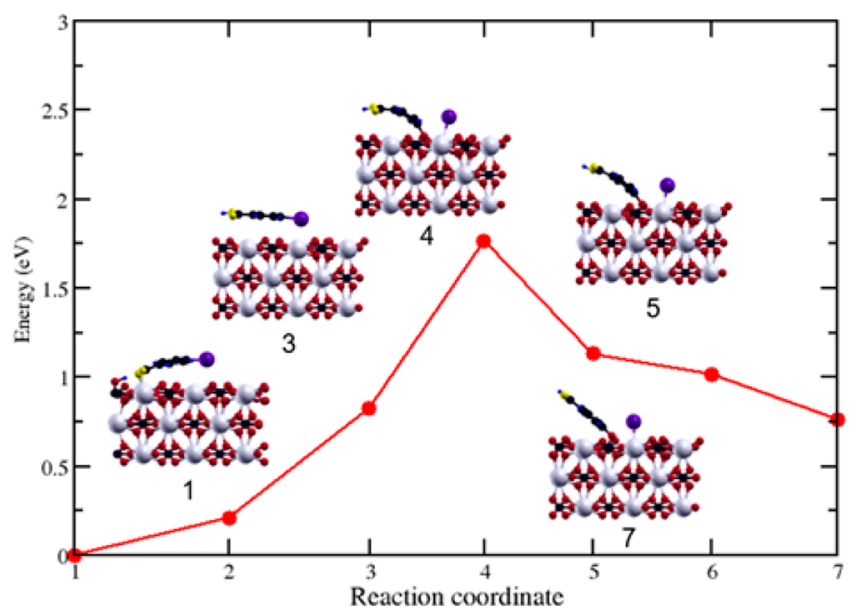

Figure 6. Minimum-energy profile and reaction path for a single dehalogenation process involving one IBA molecule flat-lying on calcite (10.4). The transition between the IS (Figure 3b) and FS (Figure $3 \mathrm{e}$ ) has an energy barrier of $1.76 \mathrm{eV}$. The barrier is reduced with respect to the gas phase through the stabilization effect achieved by the de-Ha IBA chemisorption and by the iodine adsorption in the FS. Note that, for a process having an upright IBA as its IS (not shown), a barrier similar to that in the gas phase is expected $(3.19 \mathrm{eV})$, as the iodine would first have to go into the gas phase.

considered, including the gain in entropy. Here, the major contribution comes from the diatomic gas formed upon $\mathrm{I}_{2}$ desorption. We estimate this contribution to be in the range from 2.6 to $3.2 \mathrm{eV}$ per $\mathrm{I}_{2}$ molecule for the temperature interval of $500-600 \mathrm{~K}$ (section S11). This high contribution makes the recombinative desorption of $\mathrm{I}_{2}$ thermodynamically favorable. In summary, the sequence of independent mechanism(s) described above and involving chemisorption must be considered as a possible pathway, clearly pointing to a catalytic role of the surface in lowering the rate-determining barrier associated with the dehalogenation step.

Importantly, the same $\mathrm{I}_{2}$ gas entropy gain also applies also to all FSs considered below. The main fundamental process initiating the formation of the iodine molecule is the de-Ha reaction. Hence, by comparing various de-Ha mechanisms, it should be possible to assess their likelihood. According to the classical transition-state (TS) theory in the harmonic approximation, ${ }^{56}$ the transition rate depends on the energy barrier (the difference in total energy between the TS and the minimum), the temperature, and the entropic effects. The latter basically enters through the prefactor containing the ratio of the vibrational frequencies at the minimum and at the saddle point. In most cases, the prefactor varies between $10^{12}$ and $10^{14} \mathrm{~s}^{-1}$, corresponding to the characteristic vibrational frequency "responsible" for the transition. Numerically, however, the importance of the prefactor is less serious than that of the energy barrier, which enters exponentially. This allowed us to concentrate on the calculation of energy barriers in assessing the likelihood of various transitions, while the prefactor was fixed at the value of $10^{13} \mathrm{~s}^{-1}$. Note that entropic effects due to the $I_{2}$ gas affect only the free energy of the final state and, hence, do not affect the calculated transition rates.

2.7. Processes Involving Two Flat-Lying Molecules. As a second possible mechanism, we considered two molecules that react together in a unique process. This involves a double dehalogenation with subsequent dimerization. Several cases are considered, all sharing energetically very similar ISs and the same FS. As an IS, we consider two separate flat-lying molecules facing each other at some distance along [010]. Each unreacted molecule is in a configuration similar to that shown in Figure 3b, the second most stable configuration along [010]. The FS is the stable configuration of a covalent dimer also oriented along [010], with the iodine atoms forming an iodine molecule in the gas phase (Figure 5 and section S6). This dimer is the building block of the observed chains along $[\overline{42} 1]$. The reaction is exothermic, with a total energy gain of $\Delta E_{(\mathrm{FS}-\mathrm{IS})}=-1.14 \mathrm{eV}$.

The first process is illustrated in Figure 7a, where two molecules belonging to a chain dehalogenate without chemisorption on the surface. The barrier is $2.8 \mathrm{eV}$. This value is much lower than the sum of two concomitant iodine detachments, as the NEB identifies an asynchronous pathway in which the two iodine atoms detach at different times. This is, in general, another important aspect that facilitates the reaction. As discussed above, IBA chemisorption after dehalogenation also plays an important catalytic role. In Figure $7 \mathrm{~b}$, we consider a second process where the dehalogenations are asynchronous and both phenyl radicals bind to calcite, individually realizing the most stable dehalogenated configuration on the surface (similar to Figure $3 \mathrm{~d}, \mathrm{e}$ ). The barrier is $2.8 \mathrm{eV}$, still rather high and similar to that in Figure 7a. This shows that, in the overall process involving two molecules, the IBA chemisorption has a marginal catalytic effect, even though it was found to help lower the barrier in the independent scenario involving single molecules. Interestingly, what makes this process still costly are the synchronous detachments of the phenyl radicals from the surface before dimerization, as shown in Figure $7 \mathrm{~b}$.

2.8. Two-Molecule Mechanism with Strongly Reduced Energy Barriers. It was mentioned above that the upright phase is the molecular arrangement observed just before the formation of the chains of dimers (Figure 2). Therefore, as the third possible mechanism, we considered an IS consisting of two upright molecules anchored on calcite with the carboxylate group oriented along [421] and placed apart along [010]. Their $\mathrm{H}$ atoms are initially detached from the respective nearest surface $\mathrm{O}$ atoms, as tentatively concluded from the experimental results. ${ }^{32}$ This geometry facilitates the formation of a dimer in the relaxed chain geometry of Figure 5a. At the same time, we believe that this process must be extremely relevant for the phase transition under discussion, as this IS (even though an upright single molecule is less stable than a flat-lying one) can be a possible geometry within the upright phase, for example, at the edge of an island or during the structural changes occurring at $580 \mathrm{~K}$. Hence, an IS made of upright molecules is likely to lead to the IBA dimer, and in our simplified scheme, the corresponding process would be one of the elementary steps of the phase transition between the upright phase and the chains of dimers.

In Figure 8, we present a rather nontrivial reaction mechanism that incorporates most of the catalyzing factors highlighted above. During the reaction (Figure 8, left panel), the cost of the dehalogenation involving the first iodine atom (i1) is reduced, as the second iodine atom (i2) is first "exchanged" and then "shared" between the two molecules. Thus, a plateau of intermediate low-energy states with both molecules attached to the same iodine atom (i2) is formed (Figure 8, right panel). Only at that point does i2 detach and join il to form the $I_{2}$ molecule, whereas the IBA molecules dimerize through the covalent linking between phenyls. The detachments give rise to two barriers whose heights (1.85 and 

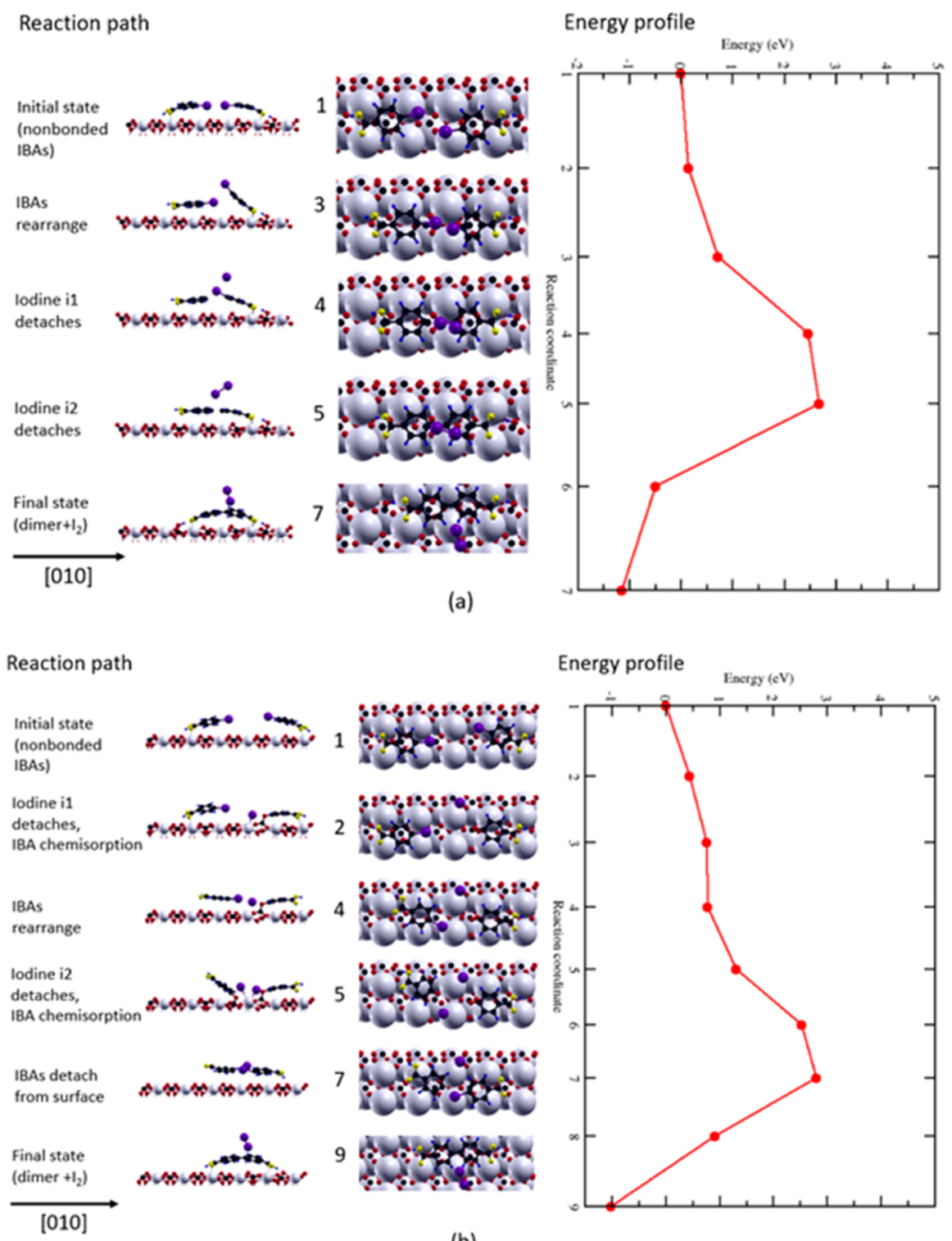

(b)

Figure 7. Reaction paths and minimum-energy profiles of two different processes involving two flat-lying molecules oriented along the [010] direction on calcite. ${ }^{55}$ In both cases, the reaction consists of two dehalogenation reactions followed by a dimerization and formation of the $\mathrm{I}_{2}$ molecule. All reactions are exothermic with the total energy gain of $\Delta E_{(\mathrm{FS}-\mathrm{IS})} \approx-1 \mathrm{eV}$. (a) Process in which the dehalogenations are asynchronous, with the first "assisted" by the second, with a barrier of $2.8 \mathrm{eV}$. (b) Process in which the de-Ha phenyl radicals bind to the surface in the intermediate states $\left(R_{\mathrm{c}}=2-5\right)$ before dimerization. The barrier is $2.8 \mathrm{eV}$, similar to that in panel a. The main peak at $R_{\mathrm{c}}=6,7$ is related to the two synchronous detachments from the surface that are required for the de-Ha IBA molecules to dimerize.

$1.5 \mathrm{eV}$ ) are dramatically reduced in comparison with those of all two-molecule processes considered above, whose barriers are $\sim 2.8 \mathrm{eV}$. Note that, in this case, the barrier for two de-Ha configurations is also far less than the sum of individual barriers (if they occurred independently in the gas phase), as each detachment is somehow assisted by the presence of the other iodine atom, in a cooperative mechanism to lower the barrier. Similar to the mechanisms presented in Figure 7, the reaction is exothermic, with an energy difference of $\Delta E_{(\mathrm{FS}-\mathrm{IS})} \approx-1 \mathrm{eV}$ (the backward barrier is $\sim 2.6 \mathrm{eV}$ ). Moreover, the reaction products are stabilized further by the $I_{2}$ gas entropic contribution discussed above. This specific lower-barrier mechanism is based on the following key factors facilitating the reaction under study: (i) two asynchronous dehalogenations, (ii) exchange and sharing of the iodine atoms during the detachments from the molecules, and (iii) $\mathrm{I}_{2}$ recombination.
We note that the mechanism in Figure 8 implies a barrier reduction even if the molecules are considered, in the gas phase, with their carboxylic groups kept in fixed positions. The corresponding calculated barrier is $1.9 \mathrm{eV}$ (see section S9), only slightly higher than that for the process on the substrate. However, the presence of the calcite substrate is still crucial as a two-dimensional support providing the conditions for the reaction to occur, namely, the possibility for the molecules to stand upright and well anchored to the surface (see also the discussion of the dense phase in ref 43). These conditions, we emphasize, would be hardly realized in the three-dimensional gas phase, making the reaction very unlikely.

Finally, it was speculated ${ }^{4,19}$ that the cleavage of the iodinephenyl bond could be facilitated by the deprotonation of the IBA carboxylic acid group on calcite (reinterpreted as H-bond formation ${ }^{43}$ ). This interesting idea is based on the consid- 
Reaction path

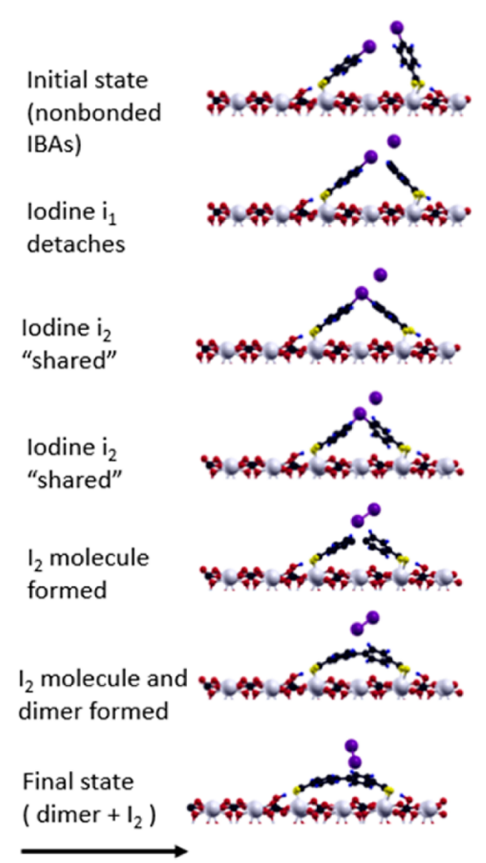

Energy profile

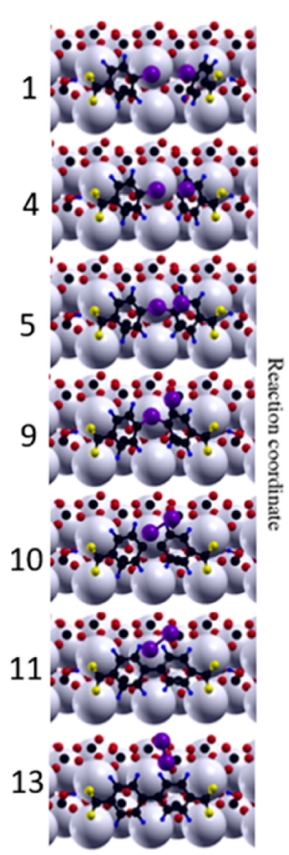

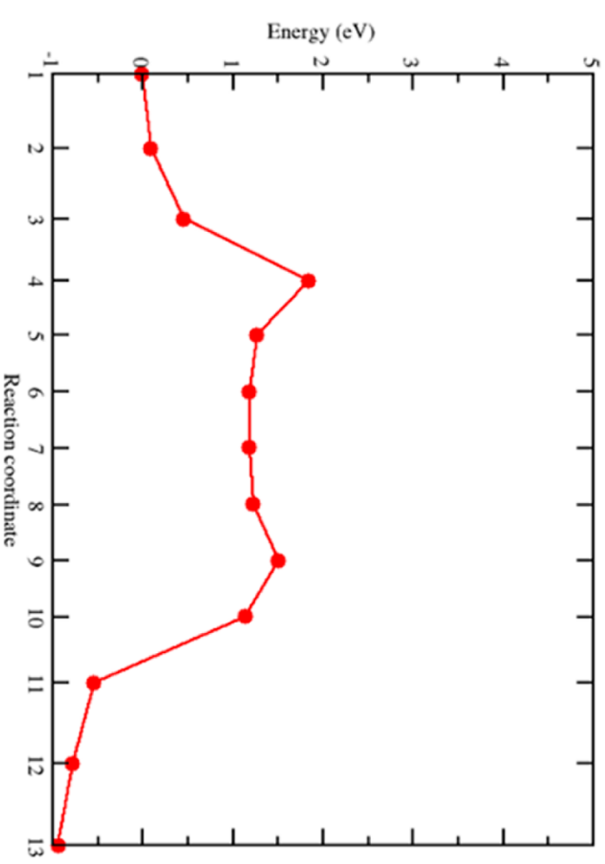

[010]

Figure 8. Reaction path and minimum-energy profile of the most favorable process of double dehalogenation and dimerization of two IBA molecules on calcite $(10.4)$. The initial state $\left(R_{c}=1\right)$ shows two upright nonbonded molecules. The first barrier $\left(R_{c}=4\right)$ is due to the dehalogenation involving the first iodine (i1), followed by a sequence of states that assist this process (reduce its energy cost) and result in the second iodine (i2) to first exchange $\left(R_{c}=5\right)$ and then become shared between the molecules $\left(R_{c}=6-9\right)$. The second barrier $\left(R_{c}=9-10\right)$ stems from the second dehalogenation, assisted by the immediate $I_{2}$ formation $\left(R_{c}=10\right)$ and the covalent dimerization $\left(R_{c}=11-13\right)$. The energy gain relative to the initial state is $\sim 1 \mathrm{eV}$, indicating the exothermic character of the reaction.

eration that the molecule, in a negative state of charge, would "lose" the iodine atom more easily. We performed a detailed analysis to address this idea. We compared the dissociation energies from a "deprotonated" IBA molecule and from a neutral molecule in the gas phase. We also compared the de-Ha energies starting from configurations on the surface in Figure 4a (where the $\mathrm{cg}-\mathrm{H}$ is attached to the molecule) and Figure $4 \mathrm{~b}$ (where the H-bond is realized). Our calculations do not support the proposed idea: We found that, in the gas phase, the de-Ha energy from a neutral molecule is the smallest $(3.19 \mathrm{eV}$; see Table S1), whereas on the surface, the de-Ha energies from the structures in panels a and b of Figure 4 are very similar ( 3.19 and $3.20 \mathrm{eV}$, respectively) and essentially equal to the gas-phase value.

\section{CONCLUSIONS}

In this work, we employed ab initio theory and NC-AFM experiments to analyze the formation mechanisms of covalently linked molecular dimers that assemble in one-dimensional chains on an insulating calcite (10.4) substrate. The dimers are made of 4-iodobenzoic acid (IBA) organic molecules, covalently connected by a (double) dehalogenation reaction. Realization of this process is nominally very costly in the gas phase at the temperatures at which the formation of this structure on calcite takes place. Focusing on this reaction and on the subsequent dimerization process, we used density functional theory and the nudged elastic band method to investigate in detail several relevant reaction paths and identify the key factors that allow these processes to occur on the substrate, with the resulting covalent assembly and chain formation.

The central result of our work is the identification of two very different processes whereby the dehalogenation barrier is strongly reduced with respect to the case of a single molecule in the gas phase $(3.19 \mathrm{eV})$, as a result of the catalytic role of the surface. The first involves single molecules that dehalogenate independently and lower the barrier $(1.76 \mathrm{eV})$ by chemisorbing on the surface upon losing the iodine atom. The second is a nontrivial and distinct reaction mechanism in which an assisted double dehalogenation followed by $\mathrm{I}_{2}$ formation and IBA dimerization is the minimum-energy path. During the process, the two iodines dehalogenate in an asynchronous way and cooperate with each other to optimize the cost of their molecular detachment prior to dimerization. Also, this specific mechanism allows a dramatic reduction of the energy barrier to $1.85 \mathrm{eV}$. We found that the surface does not play a direct role in this specific mechanism and in facilitating the reaction involving two IBA molecules. However, it provides suitable sites for molecule-substrate anchoring (through $\mathrm{Ca}-\mathrm{O}$ bonds), which is necessary for the formation of the upright phase from which the dimer chains are formed. The surface thus acts as a twodimensional support necessary to initiate the nontrivial process.

To the best of our knowledge, this is the first theoretical study of covalent dimerization mechanisms on insulators. The identification of such mechanisms helps to realize robust and stable molecular architectures electronically decoupled from the substrate, which makes them particularly suitable for chargetransport applications. It is also an important step toward increasing the molecule/substrate combinations that allow a 
proper functionalization of bulk insulating surfaces with covalent networks, a very challenging task to date.

\section{COMPUTATIONAL METHODS}

Density functional theory (DFT) and nudged elastic band (NEB) calculations were performed with the planewavepseudopotential package Quantum ESPRESSO, ${ }^{47}$ using Ultrasoft pseudopotentials ${ }^{48}$ with a wave function (charge) energy cutoff of $408 \mathrm{eV}(4080 \mathrm{eV})$ and a generalized gradient approximation-Perdew-Burke-Ernzerhof (GGA-PBE) ${ }^{49}$ exchange-correlation functional. The Grimme-D2 van der Waals interaction ${ }^{50}$ was included. All calculations presented, including those with the substrate, were performed using spin-resolved DFT. Brillouin-zone sampling included the $k=\Gamma$ point only. The calcite (10.4) substrate was modeled with a periodically repeated slab of three layers, allowing a vacuum gap between the adsorbed molecular species and the bottom layer of the slab replica of $\sim 20 \AA$. Molecule atoms and surface atoms belonging to the first two layers were allowed to relax. Forces were relaxed up to $0.026 \mathrm{eV} / \AA$, with a $1.36 \times 10^{-7} \mathrm{eV}$ cutoff on the total energy. A smearing of $1.36 \times 10^{-2} \mathrm{eV}$ was used to improve convergence in the electronic iterations. Energy and force parameters were chosen to ensure that the calculations were well-converged. Convergence of NEB processes with respect to the number of images was checked in a few cases, and the number of images used was sufficient in all checked cases. The climbing image method ${ }^{51}$ was used in all NEB calculations.

\section{EXPERIMENTAL METHODS}

The experiments were performed under ultrahigh-vacuum (UHV) conditions with a base pressure typically better than $10^{-10}$ mbar. Optical-quality calcite $\left(\mathrm{CaCO}_{3}\right)$ crystals (Korth Kristalle $\mathrm{GmbH}$, Kiel, Germany) were cleaved in situ prior to the deposition of 4-iodobenzoic acid (IBA; Aldrich, Munich, Germany). Subsequently, molecularly resolved images were taken with an Omicron Nanotechnology (Taunusstein, Germany) variable-temperature atomic force microscope, operated in frequency-modulation noncontact (NC) mode. Further details can be found in ref 4 .

\section{ASSOCIATED CONTENT}

\section{S Supporting Information}

The Supporting Information is available free of charge on the ACS Publications website at DOI: 10.1021/acs.jpcc.7b02687.

(1) IBA molecule in the gas phase: structure and dehalogenation processes. (2) IBA adsorption geometries on calcite (10.4). (3) Dehalogenated IBA molecule with a iodine atom on calcite (10.4). (4) Iodine atom and $\mathrm{I}_{2}$ molecule adsorption geometries on calcite (10.4). (5) H-bond energy. (6) IBA dimer adsorption geometries on calcite (10.4). (7) Singlemolecule dehalogenation on calcite (10.4). (8) Dehalogenation process with $\mathrm{I}_{2}$ molecule formation. (9) Double dehalogenation-dimerization process in the gas phase. (10) Two nonbonded IBA molecules adsorption geometries on calcite (10.4). (11) Entropy contribution of a gas of $I_{2}$ molecules to the free energy. (12) Spin-resolved versus spin-unresolved energy barriers in processes including the substrate. (13) Stable configurations of two hydrogen atoms in the presence of a calcite (10.4) surface (PDF)

\section{AUTHOR INFORMATION}

\section{Corresponding Authors}

*Phone: +44 152283 5884. E-mail: afloris@lincoln.ac.uk.

*Phone: +44 0207848 2160. E-mail: lev.kantorovitch@kcl.ac. uk.

*Phone: +86 1587135 1027. E-mail: yu.wang@whu.edu.cn.

ORCID $\odot$

Angelika Kühnle: 0000-0003-1214-1006

Andrea Floris: 0000-0002-3160-6676

\section{Notes}

The authors declare no competing financial interest.

\section{ACKNOWLEDGMENTS}

C.G. and Y.W. gratefully acknowledge financial support from the National Natural Science Foundation of China under Grant 11574238. A.F. and L.K. acknowledge funding from an EPSRC grant (EP/J019844/1). Through our membership in the UK's HEC Materials Chemistry Consortium, which is funded by EPSRC (EP/L000202), this work used the ARCHER UK National Supercomputing Service (http://www.archer.ac.uk). A.K. gratefully acknowledges financial support from the EU through Grant PAMS (seventh framework program GA 610446). C.G., A.F., and L.K. thank Chiara Paris for help in providing some useful material for the realization of this work.

\section{REFERENCES}

(1) Matena, M.; Riehm, T.; Stöhr, M.; Jung, T. A.; Gade, L. H. Transforming surface coordination polymers into covalent surface polymers: Linked polycondensed aromatics through oligomerization of N-heterocyclic carbene Intermediates. Angew. Chem., Int. Ed. 2008, $47,2414-2417$.

(2) Grill, L.; Dyer, M.; Lafferentz, L.; Persson, M.; Peters, M. V.; Hecht, S. Nano-architectures by covalent assembly of molecular building blocks. Nat. Nanotechnol. 2007, 2, 687-691.

(3) Rahe, P.; Kittelmann, M.; Neff, J. L.; Nimmrich, M.; Reichling, M.; Maass, P.; Kühnle, A. Tuning molecular self-assembly on bulk insulator surfaces by anchoring of the organic building blocks. Adv. Mater. 2013, 25, 3948-3956.

(4) Kittelmann, M.; Rahe, P.; Nimmrich, M.; Hauke, C. M.; Gourdon, A.; Kühnle, A. On-surface covalent linking of organic building blocks on a bulk insulator. ACS Nano 2011, 5, 8420-8425.

(5) Floris, A.; Haq, S.; In't Veld, M.; Amabilino, D. B.; Raval, R.; Kantorovich, L. Driving forces for covalent assembly of porphyrins by selective $\mathrm{C}-\mathrm{H}$ bond activation and intermolecular coupling on a copper surface. J. Am. Chem. Soc. 2016, 138, 5837-5847.

(6) Lindner, R.; Kühnle, A. On-Surface Reactions. ChemPhysChem 2015, 16, 1582-1592.

(7) In't Veld, M.; Iavicoli, P.; Haq, S.; Amabilino, D. B.; Raval, R. Unique intermolecular reaction of simple porphyrins at a metal surface gives covalent nanostructures. Chem. Commun. 2008, 1536-1538.

(8) Björk, J. Reaction mechanisms for on-surface synthesis of covalent nanostructures. J. Phys.: Condens. Matter 2016, 28, 083002.

(9) Abdel-Mottaleb, M. M. S.; Gomar-Nadal, E.; Surin, M.; Uji-I, H.; Mamdouh, W.; Veciana, J.; Lemaur, V.; Rovira, C.; Cornil, J.; Lazzaroni, R.; Amabilino, D. B.; De Feyter, S.; De Schryver, F. C. Selfassembly of tetrathiafulvalene derivatives at a liquid/solid interfacecompositional and constitutional influence on supramolecular ordering. J. Mater. Chem. 2005, 15, 4601-4615.

(10) Puigmartí-Luis, J.; Minoia, A.; Uji-I, H.; Rovira, C.; Cornil, J.; De Feyter, S.; Lazzaroni, S.; Amabilino, D. B. Noncovalent control for bottom-up assembly of functional supramolecular wires. J. Am. Chem. Soc. 2006, 128, 12602-12603.

(11) Abdurakhmanova, N.; Floris, A.; Tseng, T. C.; Comisso, A.; Stepanow, S.; De Vita, A.; Kern, K. Stereoselectivity and electrostatics 
in charge-transfer $\mathrm{Mn}$ - and $\mathrm{Cs}-\mathrm{TCNQ}_{4}$ networks on $\mathrm{Ag}(100)$. Nat. Commun. 2012, 3, 940.

(12) Floris, A.; Comisso, A.; De Vita, A. Fine-tuning the electrostatic properties of an alkali-linked organic adlayer on a metal substrate. ACS Nano 2013, 7, 8059-8065.

(13) Blüm, M.-C.; Ćavar, E.; Pivetta, M.; Patthey, F.; Schneider, W.D. Conservation of chirality in a hierarchical supramolecular selfassembled structure with pentagonal symmetry. Angew. Chem., Int. Ed. 2005, 44, 5334-5337.

(14) Tomba, G.; Stengel, M.; Schneider, W.-D.; Baldereschi, A.; De Vita, A. Supramolecular self-assembly driven by electrostatic repulsion: The $1 \mathrm{D}$ aggregation of rubrene pentagons on $\mathrm{Au}(111)$. ACS Nano 2010, 4, 7545-7551.

(15) Della Pia, A.; Riello, M.; Floris, A.; Stassen, D.; Bonifazi, D.; Jones, T. S.; De Vita, A.; Costantini, G. Anomalous coarsening driven by reversible charge transfer at metal organic interfaces. ACS Nano 2014, 8, 12356-12364.

(16) Willner, A. E.; Byer, R. L.; Chang-Hasnain, C. J.; Forrest, S. R.; Kressel, H.; Kogelnik, H.; Tearney, G. J.; Townes, C. H.; Zervas, M. N. Optics and Photonics: Key Enabling Technologies. Proc. IEEE 2012, 100, 1604-1643.

(17) Nilius, N.; Risse, T.; Schauermann, S.; Shaikhutdinov, S.; Sterrer, M.; Freund, H.-J. Model studies in catalysis. Top. Catal. 2011, $54,4-12$.

(18) Abel, M.; Clair, S.; Ourdjini, O.; Mossoyan, M.; Porte, L. Single layer of polymeric fe-phthalocyanine: An organometallic sheet on metal and thin insulating film. J. Am. Chem. Soc. 2011, 133, 12031205

(19) Kittelmann, M.; Nimmrich, M.; Lindner, R.; Gourdon, A.; Kühnle, A. Sequential and site-specific on-surface synthesis on a bulk insulator. ACS Nano 2013, 7, 5614-5620.

(20) Lindner, R.; Rahe, P.; Kittelmann, M.; Gourdon, A.; Bechstein, R.; Kühnle, A. Substrate Templating Guides Photo-Induced Reaction of C60 on Calcite. Angew. Chem., Int. Ed. 2014, 53, 7952-7955.

(21) Haq, S.; Hanke, F.; Sharp, J.; Persson, M.; Amabilino, D. B.; Raval, R. Versatile bottom-up construction of diverse macromolecules on a surface observed by scanning tunneling microscopy. ACS Nano 2014, 8, 8856-8870.

(22) Lafferentz, L.; Eberhardt, V.; Dri, C.; Africh, C.; Comelli, G.; Esch, F.; Hecht, S.; Grill, L. Controlling on-surface polymerization by hierarchical and substrate-directed growth. Nat. Chem. 2012, 4, 215220.

(23) Cai, J. M.; Ruffieux, P.; Jaafar, R.; Bieri, M.; Braun, T.; Blankenburg, S.; Muoth, M.; Seitsonen, A. P.; Saleh, M.; Feng, X. L.; et al. Atomically Precise Bottom-up Fabrication of Graphene Nanoribbons. Nature 2010, 466, 470-473.

(24) Lee, J.; Dougherty, D. B.; Yates, J. T., Jr. Chemisorbed benzoateto-benzene conversion via phenyl radicals on $\mathrm{Cu}(110)$ : kinetic observation of conformational effects. J. Am. Chem. Soc. 2006, 128, 6008-6009.

(25) Treier, M.; Pignedoli, C. A.; Laino, T.; Rieger, R.; Müllen, K.; Passerone, D.; Fasel, R. Surface-assisted cyclodehydrogenation provides a synthetic route towards easily processable and chemically tailored nanographenes. Nat. Chem. 2011, 3, 61-67.

(26) Björk, J.; Hanke, F.; Stafström, S. Mechanisms of halogen-based covalent self-assembly on metal surfaces. J. Am. Chem. Soc. 2013, 135, $5768-5775$.

(27) Haq, S.; Hanke, F.; Dyer, M. S.; Persson, M.; Iavicoli, P.; Amabilino, D. B.; Raval, R. Clean coupling of unfunctionalized porphyrins at surfaces to give highly oriented organometallic oligomers. J. Am. Chem. Soc. 2011, 133, 12031-12039.

(28) Hanke, F.; Haq, S.; Raval, R.; Persson, M. Heat-to-connect: Surface commensurability directs organometallic one-dimensional selfassembly. ACS Nano 2011, 5, 9093-9103.

(29) Björk, J.; Zhang, Y. Q.; Klappenberger, F.; Barth, J. V.; Stafström, S. Unraveling the mechanism of the covalent coupling between terminal alkynes on a noble metal. J. Phys. Chem. C 2014, 118, 3181-3187.
(30) Gao, H. Y.; Franke, J.; Wagner, H.; Zhong, D.; Held, P. A.; Studer, A.; Fuchs, H. Effect of metal surfaces in on-surface glaser coupling. J. Phys. Chem. C 2013, 117, 18595-18602.

(31) Björk, J.; Stafström, S.; Hanke, F. Zipping up: Cooperativity drives the synthesis of graphene nanoribbons. J. Am. Chem. Soc. 2011, 133, 14884-14887.

(32) Kittelmann, M.; Nimmrich, M.; Neff, J. L.; Rahe, P.; Greń, W.; Bouju, X.; Gourdon, A.; Kühnle, A. Controlled activation of substrate templating in molecular self-assembly by deprotonation. J. Phys. Chem. C 2013, 117, 23868-23874.

(33) Pawlak, R.; Nony, L.; Bocquet, F.; Oison, V.; Sassi, M.; Debierre, J. M.; Loppacher, C.; Porte, L. Supramolecular assemblies of 1,4-benzene diboronic acid on $\mathrm{KCl}(001)$. J. Phys. Chem. C 2010, 114, 9290-9295.

(34) Rahe, P.; Lindner, R.; Kittelmann, M.; Nimmrich, M.; Kühnle, A. From dewetting to wetting molecular layers: $\mathrm{C}_{60}$ on $\mathrm{CaCO}_{3}(10.4)$ as a case study. Phys. Chem. Chem. Phys. 2012, 14, 6544-6548.

(35) Kunstmann, T.; Schlarb, A.; Fendrich, M.; Wagner, T.; Möller, R.; Hoffmann, R. Dynamic force microscopy study of 3,4,9,10perylenetetracarboxylic dianhydride on $\mathrm{KBr}(001)$. Phys. Rev. B: Condens. Matter Mater. Phys. 2005, 71, 121403.

(36) Nony, L.; Bennewitz, R.; Pfeiffer, O.; Gnecco, E.; Baratoff, A.; Meyer, E.; Eguchi, T.; Gourdon, A.; Joachim, C. Cu-TBPP and PTCDA molecules on insulating surfaces studied by ultra-high-vacuum non-contact AFM. Nanotechnology 2004, 15, S91.

(37) Neff, J. L.; Kittelmann, M.; Bechstein, R; Kühnle, A. Decisive influence of substitution positions in molecular self-assembly. Phys. Chem. Chem. Phys. 2014, 16, 15437-15443.

(38) Kittelmann, M.; Rahe, P.; Gourdon, A.; Kühnle, A. Direct visualization of molecule deprotonation on an insulating surface. ACS Nano 2012, 6, 7406-7411.

(39) Kittelmann, M.; Rahe, P.; Kühnle, A. Molecular self-assembly on an insulating surface: Interplay between substrate templating and intermolecular interactions. J. Phys.: Condens. Matter 2012, 24, 354007.

(40) Hauke, C. M.; Bechstein, R.; Kittelmann, M.; Storz, C.; Kilbinger, A. F.; Rahe, P.; Kühnle, A. Controlling Molecular SelfAssembly on an Insulating Surface by Rationally Designing an Efficient Anchor Functionality That Maintains Structural Flexibility. ACS Nano 2013, 7, 5491.

(41) Rahe, P.; Nimmrich, M.; Greuling, A.; Schütte, J.; Stará, I. G.; Rybáček, J.; Huerta-Angeles, G.; Starý, I.; Rohlfing, M.; Kühnle, A. Toward molecular nanowires self-assembled on an insulating substrate: Heptahelicene-2-carboxylic acid on calcite (10.4). J. Phys. Chem. C 2010, 114, 1547-1552.

(42) Neff, J. L.; Söngen, H.; Bechstein, R.; Maass, P.; Kühnle, A. Long range order induced by intrinsic repulsion on an insulating substrate. J. Phys. Chem. C 2015, 119, 24927.

(43) Paris, C.; Floris, A.; Aeschlimann, S.; Kittelmann, M.; Kling, F.; Bechstein, R.; Kühnle, A.; Kantorovich, L. Increasing the templating effect on a bulk insulator surface: From a kinetically trapped to a thermodynamically more stable structure. J. Phys. Chem. C 2016, 120 (31), 17546-17554.

(44) Jónsson, H.; Mills, G.; Jacobsen, K. W. Nudged elastic band method for finding minimum energy paths of transitions. In Classical and Quantum Dynamics in Condensed Phase Simulations; Berne, B. J., Ciccotti, G., Coker, D. F., Eds.; World Scientific: Singapore, 1998; pp $385-404$.

(45) Lide, D. R, Ed. Bond strengths in polyatomic molecules. In CRC Handbook of Chemistry and Physics; CRC Press: Boca Raton, FL, 2005.

(46) Morchutt, C.; Björk, J.; Krotzky, S.; Gutzler, R.; Kern, K. Covalent coupling via dehalogenation on $\mathrm{Ni}(111)$ supported boron nitride and graphene. Chem. Commun. 2015, 51, 2440-2443.

(47) Giannozzi, P.; Baroni, S.; Bonini, N.; Calandra, M.; Car, R.; Cavazzoni, C.; Ceresoli, D.; Chiarotti, G. L.; Cococcioni, M.; Dabo, I.; et al. QUANTUM ESPRESSO: A modular and open-source software project for quantum simulations of materials. J. Phys.: Condens. Matter 2009, 21, 395502. 
(48) Vanderbilt, D. Soft self-consistent pseudopotentials in a generalized eigenvalue formalism. Phys. Rev. B: Condens. Matter Mater. Phys. 1990, 41, 7892.

(49) Perdew, J. P.; Burke, K.; Ernzerhof, M. Generalized gradient approximation made simple. Phys. Rev. Lett. 1996, 77, 3865-3868.

(50) Grimme, S. Semiempirical gga-type density functional constructed with a long-range dispersion correction. J. Comput. Chem. 2006, 27, 1787-1779.

(51) Henkelman, G.; Uberuaga, B. P.; Jónsson, H. A climbing image nudged elastic band method for finding saddle points and minimum energy paths. J. Chem. Phys. 2000, 113, 9901-9904.

(52) For more clarity, we denote by "chemisorption" only the process in which a bond between the phenyl radical and an oxygen of the surface is realized after the dehalogenation.

(53) A rough estimate of the temperature necessary for this reaction to occur can be obtained by assuming a simple Arrhenius law. Taking an attempt frequency of $10^{12} \mathrm{~s}^{-1}$, the energy barrier of $\sim 3 \mathrm{eV}$ corresponds to a temperature of nearly $1000 \mathrm{~K}$ when one assumes that one-half of the molecules are dissociated after $30 \mathrm{~min}$. This is surely way more than the experimentally required temperature.

(54) This is corroborated by our NEB calculations showing, in some cases, that the $\mathrm{H}$ atom shuttles during the process, indicating that a very low energy barrier must exist between the two states. Note also that the effects of the $\mathrm{H}$ position on the dehalogenation and dimerization barriers are rather marginal.

(55) We do not define the reaction coordinate here, as it is given by a linear combination of many atomic degrees of freedom to indicate the direction to the transition state. Such a definition can be meaningful only within a narrow vicinity of each point along the minimum-energy path. This approach is sufficient for our purposes given that we are exclusively interested in the barriers associated with different paths. In this case, it is also possible to show several paths on one graph even though they do not correspond to the same reaction coordinates.

(56) Vineyard, G. H. Frequency factors and isotope effects in solid state rate processes. J. Phys. Chem. Solids 1957, 3, 121-127. 